\title{
Ventana Pericardica: Abordaje Subxifoideo vs Toracoscopico En Trauma Precordial Abierto
}

\author{
${ }^{1}$ Laura J Delgado Mateus, ${ }^{2}$ Ada M Bustos Guerrero, ${ }^{3}$ Andrés G Barco Manrique, ${ }^{4}$ Juan P Serrano Pastrana \\ ${ }^{5}$ Oscar F Herrán Falla, ${ }^{6}$ Laura I Valencia-Ángel
}

\section{RESUMEN}

Introducción: La ventana pericárdica es el estándar de oro en el diagnóstico de trauma cardiaco en heridas precordiales estando descritas diferentes vías de abordaje. El objetivo de este estudio es comparar las características clínicas, desenlaces y rendimiento diagnóstico de las vías subxifoidea vs. toracoscópica en pacientes con trauma precordial abierto.

Diseño del Estudio: Estudio observacional de cohortes, en un periodo de 56 meses, se recopilaron pacientes con heridas penetrantes en región precordial, hemodinámicamente estables que ingresaron al Servicio de Urgencias, a quienes se indicó la realización de ventana pericárdica para descartar lesiones cardiacas. Se compararon las características clínicas, desenlaces y el rendimiento diagnóstico de las pruebas.

Resultados: Se incluyeron 256 pacientes, 93.4\% ingresaron por herida por arma cortopunzante, $18.8 \%$ de las ventanas pericárdicas fueron vía toracoscópica, $20.8 \%$ resultaron positivas para hemopericardio y $11.5 \%$ de las subxifoideas, la mediana de la estancia hospitalaria fue 4 días, $16.0 \%$ presentaron complicaciones, $17.8 \%$ en pacientes abordados por vía subxifoidea y $8.3 \%$ vía toracoscópica siendo más frecuente el hemotórax coagulado, 15.2\% fueron llevados a reintervención, la mayoría a toracoscopia para drenaje de hemotórax coagulado $(62.5 \%)$, la mortalidad fue $1.6 \%$, todos los casos en el

\footnotetext{
${ }^{1}$ Médica y Cirujana, Residente de Cirugía General, ${ }^{2}$ Médica General, ${ }^{3}$ Cirujano General, ${ }^{4}$ Cirujano General, Profesor ${ }^{5}$ Nutricionista y Dietista, Magíster en Epidemiología, ${ }^{6}$ Médica y Cirujana, Magíster en Epidemiología

${ }^{1,6}$ Departamento de Cirugía General, Universidad Industrial de Santander, Hospital Universitario de Santander, Bucaramanga Colombia

${ }^{2}$ Servicio de Cirugía de Tórax, Clínica Chicamocha, Bucaramanga Colombia

${ }^{3}$ Departamento de Cirugía General, Clínica Carlos Ardila Lülle Floridablanca, Colombia

${ }^{4}$ Departamento de Cirugía, Universidad Industrial de Santander Bucaramanga, Colombia

${ }^{5}$ Escuela de Escuela de Nutrición y Dietética. Centro de Investigaciones Epidemiológicas, Universidad Industrial de Santander, Bucaramanga, Colombia

Corresponding Address: Ada M Bustos Guerrero, Médica General, Servicio de Cirugía de Tórax, Clínica Chicamocha Bucaramanga, Colombia, teléfono: +573017596026 , e-mail: adabustosmd@gmail.com
}

grupo del abordaje subxifoideo. La sensibilidad de la ventana pericárdica subxifoidea fue $95.8 \%$ y la toracoscópica $90.9 \%$, y la especificidad fue $99.5 \%$ y $100 \%$, respectivamente.

Conclusión: El abordaje toracoscópico es una opción diagnóstica con rendimiento similar al subxifoideo. A pesar de establecer una disminución en la mediana de los días como usuarios de sonda de toracostomía, no se observó disminución en los días de estancia hospitalaria.

Palabras claves: Abordaje subxifoideo, Abordaje toracoscópico, Heridas precordiales, Trauma precordial abierto, Ventana pericárdica.

How to cite this article: Mateus LJD, Guerrero AMB, Manrique AGB, Pastrana JPS, Falla OFH, Valencia-Ángel LI. Ventana Pericardica: Abordaje Subxifoideo vs. Toracoscopico En Trauma Precordial Abierto. Panam J Trauma Crit Care Emerg Surg 2017;6(3):135-141.

Source of support: Nil

Conflict of interest: None

\section{ABSTRACT}

Introduction: The pericardial window is the gold standard in the diagnosis of cardiac trauma in precordial wounds and different approaches are described. The aim of this study is to compare the clinical characteristics, outcomes, and diagnostic performance of subxiphoid vs. thoracoscopy in patients with open precordial trauma.

Study design: A 56-month observational study of cohorts collected patients with penetrating wounds in the precordial region was conducted. These patients were hemodynamically stable and admitted to the Emergency Department. They were asked to perform a pericardial window to rule out cardiac lesions. The clinical characteristics, outcomes, and diagnostic performance of the tests were compared.

Results: A total of 256 patients were included in the study. Around $93.4 \%$ were injured by a sharp gunshot wound, $18.8 \%$ of the pericardial windows were thoracoscopic, $20.8 \%$ were positive for hemopericardium, and $11.5 \%$ were subxiphoid. The median length of hospital stay was 4 days, we had $16.0 \%$ complications, $17.8 \%$ in subxiphoid approach, and $8.3 \%$ in thoracoscopic patients, with coagulated hemothorax being the most frequent, $15.2 \%$ were submitted to reintervention, most of them thoracoscopy for clotted hemothorax drainage $(62.5 \%)$, mortality was $1.6 \%$, all cases in the subxiphoid group. The sensitivity of the subxiphoid pericardial window was $95.8 \%$ and thoracoscopic $90.9 \%$, and the specificity was $99.5 \%$ and $100 \%$ respectively.

Este trabajo fue llevado a cabo en el Hospital Universitario de Santander, Bucaramanga, Colombia

Este trabajo fue presentado en Maceió - Brasil, en el XXIX Congreso Panamericano de trauma, cuidado crítico y emergencias quirúrgicas 
Conclusion: The thoracoscopic approach is a diagnostic option with subxiphoid-like performance. In spite of establishing a decrease in the median of the days as users of thoracostomy tube, no decrease was observed in the days of hospital stay.

Keywords: Open precordial trauma, Pericardial window, Precordial wounds, Subxiphoid approach, Thoracoscopic approach.

\section{INTRODUCCIÓN}

El trauma penetrante cardiaco es un reto frente al cual se enfrentan los equipos quirúrgicos del mundo en su práctica diaria. Es una lesión altamente letal correspondiendo al $0.1 \%$ de las admisiones por trauma; las estadísticas evidencian que el $80-90 \%$ de los pacientes que sufren heridas cardiacas penetrantes fallecen antes de la atención médica, ${ }^{1-3}$ sin embargo, en centros que cuentan con un sistema de transporte medico organizado, hasta el $45 \%$ logran llegar al servicio de urgencias con signos vitales ${ }^{4-6}$ con una tasa de supervivencia que oscila entre $60-80 \%{ }^{2}$ En nuestro medio, no existen datos nacionales acerca de la incidencia del trauma cardiaco penetrante; Villegas et al, ${ }^{7}$ reporta que aproximadamente un $90 \%$ de los casos de heridas cardiacas en Colombia se presentan en pacientes de sexo masculino y solo un $10 \%$ en femenino, datos que son comparables con el resto de la literatura mundial. ${ }^{8}$

Las lesiones penetrantes cardiacas son principalmente causadas por armas corto-punzantes y armas de fuego, ${ }^{9}$ la relación entre los dos mecanismos es 1:2 generalmente, dependiendo del acceso que tenga la población a cada tipo de arma. En países desarrollados, es más común el uso de armas de fuego, sin embargo, en países en desarrollo como Colombia, es mayor el uso de las armas cortopunzantes hasta un $88 \%$ (en series nacionales), siendo este el mecanismo más frecuente. ${ }^{2,7}$

El diagnostico de heridas cardiacas requiere un alto índice de sospecha. El examen físico es crucial para sospechar la lesión y clasificar al paciente; la evaluación y manejo inicial debe ser realizado según el protocolo del Advanced Trauma Life Support (ATLS). Pacientes que ingresan al servicio de urgencias in extremis, con inestabilidad hemodinámica o en paro cardiorrespiratorio presenciado posterior a una herida precordial penetrante y que sean potencialmente salvables, deben ser llevados a toracotomía de reanimación inmediatamente. ${ }^{3}$ En pacientes estables hemodinámicamente (PAS $>90 \mathrm{~mm} \mathrm{Hg}$ o PAM > $60 \mathrm{mmHg}$ ), existen métodos diagnósticos que ayudan al evaluador a detectar o descartar una lesión cardiaca; actualmente el FAST y eFAST son métodos seguros no invasivos, rápidos y se pueden hacer en el servicio de urgencias al ingreso, sin embargo es operador dependiente y no todas las instituciones cuentan con este método diagnóstico disponible 24 horas al día. La ventana pericárdica es el estándar de oro en el diagnóstico de trauma cardiaco en heridas precordiales en este tipo de pacientes. ${ }^{3}$

Se han descrito diferentes vías de abordaje, transtoracica (toracoscopia/toracotomía/esternotomia) vs transabdominal (subxifoidea o transdiafragmatica). La via toracoscópica tiene la ventaja de realizar tratamietn de otras lesiones intratoracicas, al igual que la via transdiafragmatica por laparoscopia puede ser útil en el diagnostico de otras heridas de la cavidad peritoneal en los pacientes con heridas en la región toracoabdominal. ${ }^{10-12}$ Sin embargo, en el presente estudio únicamente se tomaron en cuenta las dos vías de abordaje mas usadas: Toracoscopica vs Subxifoidea.

\section{MATERIALES Y MÉTODOS}

Se trata de un estudio observacional de cohortes en el que se identificaron todos los pacientes que ingresaron al Servicio de Urgencias de un Hospital de tercer nivel de complejidad en el Nororiente Colombiano entre enero de 2012 y agosto de 2016 y que fueron llevados a pericardiotomía. Se incluyeron en el análisis los pacientes con heridas penetrantes de la región precordial que ingresaron hemodinámicamente estables y llevados a ventana pericárdica para descartar lesiones cardiacas por vía toracoscópica o subxifoidea. Los individuos que fueron llevados a otras vías de abordaje fueron excluidos. La región precordial se definió como el área comprendida por debajo de las clavículas y por encima del reborde costal incluyendo el epigastrio, entre la línea axilar anterior izquierda y la línea medio clavicular derecha.

Se recolectaron datos demográficos y clínicos a través de la revisión de la historia clínica electrónica utilizando un formato creado con este fin y posteriormente se digitaron usando un formulario de Google Drive ${ }^{\circledR}$ por duplicado, se validaron las bases de datos usando la herramienta validate de EpiData ${ }^{\circledR}$ hasta lograr dos bases de datos idénticas que fueron exportadas en formato .dta para ser analizadas en Stata ${ }^{\circledR}$ 11.0. Las variables cuantitativas se resumieron en términos de mediana y rangos intercuartílicos y las variables cualitativas como frecuencias absolutas y relativas. Se realizó un análisis bivariado según el tipo de abordaje comparando las medianas de los dos grupos con métodos no paramétricos y las diferencias de proporciones con el test $\mathrm{Chi}^{2}$ o exact de Fisher. El método estándar para el diagnóstico de heridas cardiacas fue la toracotomía o esternotomía

La información recolectada se identificó con un número consecutivo descartando los números de identificación de los casos como mecanismo para asegurar la confidencialidad. El estudio se llevo a cabo con el aval del Comité de ética de la Universidad Industrial de Santander. 


\section{RESULTADOS}

Durante el período de observación se realizaron 326 ventanas pericárdicas, 70 sujetos no fueron incluidos en el análisis por haber sido abordados por vías diferentes a las de interés (Ej. Toracotomía, esternotomía, transdiafragmática), por indicación no traumática (Ej. Derrame pericárdico) o por pérdida de la información por cambio de número de identificación del paciente, incluyendo un total de 256 individuos en el estudio.

La mayoría de pacientes estudiados fueron hombres (94.1\%), la mediana de edad fue 26 años (RIC 21-35), la procedencia fue urbana en 238 pacientes $(93.0 \%)$, rural en $7(2.7 \%)$ y desconocida en $11(4.3 \%)$. El principal mecanismo de lesión fue el cortopunzante en 239 pacientes $(93.4 \%)$ seguido por proyectil de arma de fuego en $17(6.6 \%)$, la mediana del tiempo desde la lesión hasta el ingreso fue 82 minutos (RIC 15-180), 193 de los casos (75.4\%) se presentaron al Servicio de Urgencias con herida única en la región precordial y entre los 63 con lesiones asociadas, $30(46.9 \%)$ presentaron lesiones abdominales, $26(40.6 \%)$ en extremidades, 5 casos $(7.8 \%)$ con trauma cráneo-facial y los 3 pacientes restantes $(4.7 \%)$ tuvieron lesiones en dos o más regiones corporales. A 234 individuos $(91.4 \%)$ se les realizó radiografía de tórax al ingreso, evidenciando ocupación del espacio pleural en 194 (75.8\%): Hemoneumotórax en 68 (26.6\%), hemotórax en $67(26.2 \%)$, neumotórax en $55(21.5 \%)$ y ensanchamiento mediastinal en 4 pacientes (1.6\%).

La mediana del tiempo desde el ingreso hasta el traslado al quirófano fue 256.5 minutos (RIC 142.5 - 503.5), el abordaje de la ventana pericárdica fue subxifoideo en 208 sujetos $(81.3 \%$ ) y el resultado de la prueba fue negativo para hemopericardio en $222(86.7 \%)$ del total de procedimientos. El análisis bivariado de estas características de acuerdo al procedimiento diagnóstico realizado y su significancia estadística se presentan en la Tabla 1.

Del total de pacientes, 35 (13.7\%) presentaron lesión intrapericárdica, 19 (54.3\%) fueron heridas de ventrículo derecho, 6 (17.1\%) de ventrículo izquierdo, 4 (11.4\%) de aurícula derecha, $3(8.6 \%)$ de pericardio, $1(2.9 \%)$ de arteria pulmonar, $1(2.9 \%)$ de aorta y $1(2.9 \%)$ de arteria coronaria. A 228 (89.1\%) pacientes se les realizó toracostomía cerrada con una mediana de 4 días (RIC 2-7) hasta el retiro.

La mediana de estancia hospitalaria fue 4 días (RIC 2-7), con una estancia máxima de 40 días, se presentaron

Tabla 1: Variables demográficas y clínicas por tipo de abordaje. Ventana pericárdica: abordaje subxifoideo vs toracoscópico en trauma precordial abierto, Hospital Universitario de Santander, 2012-2016

\begin{tabular}{|c|c|c|c|}
\hline \multirow[b]{2}{*}{ Variables } & \multicolumn{2}{|c|}{ Tipo de abordaje } & \multirow[b]{2}{*}{$P^{\mathrm{a}, \mathrm{b}}$} \\
\hline & Subxifoideo & Toracoscópico & \\
\hline Sexo, n (\%) & & & 0.492 \\
\hline Hombre & $197(94.7)$ & $44(91.7)$ & \\
\hline Mujer & $11(5.3)$ & $4(8.3)$ & \\
\hline Edad, mediana (RIC) & $26(20-34)$ & $27(21-35.5)$ & 0.271 \\
\hline Procedencia, n (\%) & & & 0.099 \\
\hline Urbana & $196(94.2)$ & $42(87.5)$ & \\
\hline Rural & $4(1.9)$ & $3(6.3)$ & \\
\hline Sin información & $8(3.9)$ & $3(6.3)$ & \\
\hline Mecanismo, n (\%) & & & 0.210 \\
\hline Corto-punzante & $192(92.3)$ & $47(97.9)$ & \\
\hline Proyectil de arma de fuego & $16(7.7)$ & $1(2.1)$ & \\
\hline Tiempo al ingreso, mediana (RIC) & $60(60-180)$ & $180(60-360)$ & $<0.001$ \\
\hline Herida única en región precordial & $157(81.4)$ & $136(18.6)$ & 0.94 \\
\hline \multicolumn{4}{|l|}{ Hallazgos Rx de tórax del ingreso, $\mathrm{n}(\%)$} \\
\hline No se realizó & $22(10.6)$ & $0(0.0)$ & \\
\hline Hemoneumotórax & $56(26.9)$ & $12(25.0)$ & \\
\hline Hemotórax & $49(23.6)$ & $18(37.5)$ & \\
\hline Neumotórax & $48(23.1)$ & $7(14.6)$ & \\
\hline Normal & $32(15.4)$ & $8(16.7)$ & \\
\hline Ensanchamiento mediastinal & $1(0.5)$ & $3(1.6)$ & \\
\hline Tiempo a quirófanos, mediana (RIC) & $230(122-435.5)$ & $425(265.5-775)$ & $<0.001$ \\
\hline \multicolumn{4}{|l|}{ Resultado del procedimiento, $\mathrm{n}(\%)$} \\
\hline Positivo para hemopericardio & $24(11.5)$ & $10(20.8)$ & 0.039 \\
\hline Lesiones intrapericárdicas, n (\%) & & & 0.080 \\
\hline No & $184(88.5)$ & $37(77.1)$ & \\
\hline Cardiacas & $22(10.6)$ & $10(20.8)$ & \\
\hline Grandes vasos & $2(1.0)$ & $1(2.1)$ & \\
\hline
\end{tabular}

${ }^{\mathrm{a}}$ Test Exacto de Fischer, ${ }^{\mathrm{b}}$ Prueba de Mann-Whitney 
Tabla 2: Variables desenlace por tipo de abordaje. Ventana pericárdica: abordaje subxifoideo vs toracoscópico en trauma precordial abierto, Hospital Universitario de Santander, 2012-2016

\begin{tabular}{|c|c|c|c|}
\hline \multirow[b]{2}{*}{ Variables } & \multicolumn{2}{|c|}{ Tipo de abordaje } & \multirow[b]{2}{*}{$P^{\mathrm{a}, \mathrm{b}}$} \\
\hline & Subxifoideo & Toracoscópico & \\
\hline Toracostomía cerrada, $\mathrm{n}(\%)$ & $180(86)$ & $48(100)$ & \\
\hline Días toracostomía cerrada, mediana (RIC) & $4(2-7)$ & $3(2-4.5)$ & 0.011 \\
\hline Complicaciones, n (\%) & $37(17.8)$ & $4(8.3)$ & 0.076 \\
\hline Reintervenciones, n (\%) & $35(16.8)$ & $4(8.3)$ & 0.101 \\
\hline Días de estancia hospitalaria, mediana (RIC) & $4(2-7)$ & $3(4-5)$ & 0.439 \\
\hline Reingresos, n (\%) & $9(4.3)$ & $1(2.1)$ & 0.410 \\
\hline Mortalidad, n (\%) & $4(1.6)$ & $0(0.0)$ & \\
\hline Sensibilidad (\%) & 95.8 & 90.9 & \\
\hline Especificidad (\%) & 99.5 & 100 & \\
\hline
\end{tabular}

${ }^{\mathrm{a}}$ Test Exacto de Fischer, ${ }^{\mathrm{b}}$ Prueba de Mann-Whitney

complicaciones en 41 (16.0\%) de los casos incluyendo 29 $(11.3 \%)$ individuos que desarrollaron hemotórax coagulado, 4 (1.6\%) presentaron infección del sitio operatorio, hemotórax masivo en $3(1,2 \%)$ pacientes, $3(1.2 \%)$ casos de pericarditis, 1 caso de empiema $(0,4 \%)$, otro caso presentó además de empiema, hemotórax coagulado $(0,4 \%)$, y 1 caso con empiema, infección del sitio operatorio y mediastinitis asociada $(0.4 \%)$. No se identificaron Síndromes post-pericardiotomía. De estos pacientes, 39 (15.2\%) fueron llevados a reintervenciones, 25 (62.5\%) a toracoscopia, $6(15.0 \%)$ a toracotomía, $5(12.5 \%)$ a esternotomía, $2(5.0 \%)$ a toracostomía y $2(5.0 \%)$ a toracoscopia que requirió conversión a toracotomía.

La mortalidad durante la hospitalización fue 1.6\%, los 4 casos se presentaron en el grupo del abordaje subxifoideo y ninguno presentó lesión intrapericardica, 9 (3.5\%) pacientes fueron remitidos a otra institución de quienes se desconoce el desenlace y $243(94.9 \%)$ egresaron. Durante el período de estudio se registraron 10 (3.9\%) reingresos asociados a la lesión en región precordial y a los procedimientos realizados.

La sensibilidad de la ventana pericárdica subxifoidea fue de $95.8 \%$ y la toracoscópica $90.9 \%$, y la especificidad fue $99.5 \%$ y $100 \%$, respectivamente (Tabla 2 ).

\section{DISCUSIÓN}

En nuestro estudio evidenciamos una distribución sociodemográfica en cuanto a edad y sexo similar a la descrita por otros estudios en el área de las lesiones torácicas en la región precordial y el trauma cardiaco donde los hombres en edad productiva son los más afectados. ${ }^{7,8,11,13}$ Aunque nuestra institución cuenta con un Servicio de Trauma, centro de referencia para el nororiente del país, la mayoría de los pacientes que ingresaron al Servicio de Urgencias y que fueron incluidos en el análisis proceden de zona urbana, no contamos con datos de mortalidad de las heridas cardiacas en nuestra región para establecer si las lesiones que se presentan en regiones apartadas y las rurales tienen mayor mortalidad por la falta de acceso a atención oportuna, que sería una explicación plausible a esta tendencia. Se ha establecido en la literatura que 80 a $90 \%$ de los pacientes con herida cardiaca penetrante fallecen antes de alcanzar la atención médica ${ }^{1-3}$ y que puede disminuir hasta un $45 \%$ en lugares donde se cuenta con un sistema de trauma y atención prehospitalario óptimos, al igual que un centro de trauma certificado. ${ }^{4-6}$

El mecanismo del trauma penetrante precordial se ha descrito en países desarrollados en una razón 2:1 por heridas por proyectil de arma de fuego y por arma cortopunzante, respectivamente, ${ }^{14}$ un estudio reciente publicado por Sánchez y cols. ${ }^{8}$ observó una razón cercana a 1:1 que dista de la relación encontrada en nuestro estudio donde el mecanismo de la lesión fue arma cortopunzante en el $93.4 \%$ de los casos; siendo similar a la descrita en población Colombiana por Villegas, ${ }^{7}$ Cortés ${ }^{15}$ y Morales. ${ }^{11}$

$\mathrm{El}$ algoritmo diagnóstico de las heridas en la región precordial de nuestra institución difiere tanto en la definición del área precordial como en las ayudas diagnósticas utilizadas en otras series y guías $2,3,16,17$ dado que no contamos con ultrasonografía en el Servicio de Urgencias para la realización de FAST, llevando a casi el $100 \%$ de los pacientes con trauma penetrante de tórax en región precordial a diagnóstico por ventana pericárdica ya sea subxifoidea $(81.3 \%)$ o toracoscópica $(18.7 \%)$ hasta agosto de 2016. No se encontró evidencia de diferencia en el mecanismo de lesión de acuerdo al abordaje abierto o toracoscópico. Del total de los individuos con herida cardiaca, la mayoría presentaron lesión de ventrículo derecho, seguido de ventrículo izquierdo, pericardio y aurícula derecha de la misma manera como se ha informado en la literatura., ${ }^{2,7}$

Teníamos como hipótesis que el abordaje toracoscópico, al ser un método mínimamente invasivo, podría asociarse a un menor tiempo como usuario de toracostomía y por ende a una menor estancia hospitalaria. A través del presente estudio, observamos que el 100\% de los abordajes toracoscópicos incluyeron la implantación 
de sonda de toracostomía y que la mediana de días con el tubo a tórax es menor en este grupo (3 vs. 4 días), sin evidencia de impacto en los días de estancia hospitalaria.

Esperábamos además, una disminución en la incidencia de hemotórax coagulado en el grupo del abordaje toracoscópico dado que se ha establecido que en pacientes con hemotórax y hemoneumotórax traumáticos que son llevados a toracoscopia para drenaje del hemotórax y lavado de la cavidad pleural, la incidencia de hemotórax coagulado es menor. No se evidenciaron Síndromes post-pericardiotomìa a pesar de no contar en nuestra institución con un protocolo preventivo. El tiempo entre la lesión y el ingreso al Servicio de Urgencias; y el tiempo entre éste y el traslado a quirófano fue significativamente mayor en el grupo de pacientes llevados a ventana toracoscópica, siendo un potencial factor asociado a una incidencia de hemotórax coagulado.

Nuestra explicación del hallazgo de un tiempo prequirúrgico mayor en el grupo de ventana pericárdica toracoscópica es la decisión de llevar a los pacientes con heridas precordiales y presencia de hemotórax de varias horas de evolución a toracoscopia para drenaje del hemotórax y realización de la ventana pericárdica en un solo procedimiento. Es decir, los pacientes que no presentan sospecha de hemotórax coagulado dado el tiempo de evolución de la lesión, son llevados a Toracostomía cerrada y ventana pericárdica subxifoidea.

El rendimiento diagnóstico de los dos abordajes fue comparable, siendo la ventana pericárdica subxifoidea más sensible que la toracoscópica (95\% vs 90\%), e igualmente específica (99.5 vs $100 \%$ ), hallazgo similar al de Morales et $\mathrm{al}^{11}$ en su estudio en una población Colombiana de características demográficas similares a la nuestra. Uno de los falsos negativos de la ventana toracoscópica fue llevado a toracotomía por hemotórax masivo identificando una herida grado IV de ventrículo derecho. El segundo falso negativo fue llevado a ventana subxifoidea, se diagnosticó herida de pericardio y se interpretó como ventana negativa por no tener hemopericardio, sin embargo, la herida de pericardio corresponde a herida cardiaca grado I. Ningún paciente presentó complicaciones mortales del retraso en el diagnóstico del hemopericardio.

La mortalidad intrahospitalaria de las heridas precordiales fue del $1.6 \%$, los 4 pacientes fueron abordados por vía subxifoidea y ninguno de ellos presentó herida cardiaca o de grandes vasos intrapericárdicos, siendo esta atribuida a sepsis y otras lesiones asociadas.

\section{CONCLUSIÓN}

El abordaje toracoscópico es una opción diagnóstica con rendimiento similar al subxifoideo. A pesar de establecer una disminución en la mediana de los días como usuarios de sonda de toracostomía, no se observó una disminución en los días de estancia hospitalaria.

\section{REFERENCIAS}

1. Kamali S, Aydin MT, Akan A, Karatepe O, Sari A, Yüney E. Penetrating cardiac injury: factors affecting outcome. Turkish J Trauma Emerg Surg 2011 May;17(3):225-230.

2. Kang N, Hsee L, Rizoli S, Alison P. Penetrating cardiac injury: overcoming the limits set by Nature. Injury 2009 Sep;40(9):919-927.

3. Mattox, KL.; Moore EE.; Feliciano DV., editors. Trauma. 7th ed. New York: McGraw-Hill; 2012.

4. Thourani VH, Feliciano DV, CooperWA, Brady KM, Adams AB, Rozycki GS, Symbas PN. Penetrating cardiac trauma at an urban trauma center: a 22-year perspective. Am Surg 1999 Sep;65(9):811-818.

5. Thorson CM, Namias N, Van Haren RM, Guarch GA, Ginzburg E, Salerno TA, Schulman CI, Livingstone AS, Proctor KG. Does hemopericardium after chest trauma mandate sternotomy? J Trauma Acute Care Surg 2012 Jun;72(6):1518-1524.

6. Tyburski JG, Astra L, Wilson RF, Dente C, Steffes C. Factors affecting prognosis with penetrating wounds of the heart. J Trauma 2000 Apr;48(4):587-590.

7. Villegas M, Morales C, Rosero E, Benítez G, Cano F, Fernández I, López M, Ramírez L, Bermúdez L. Trauma cardíaco penetrante: factores pronósticos. Rev Colomb Cirugía. 2007;22(3):148-56.

8. Sánchez ÁI, García AF, Velsquez M, Puyana JC. Predictors of positive subxiphoid pericardial window in stable patients with penetrating injuries to the precordial region. Panam J Trauma Crit Care Emerg Surg 2016 Jan-Apr;5(1):43-51.

9. Wall MJ, Mattox KL, Chen CD, Baldwin JC. Acute management of complex cardiac injuries. J Trauma 1997 May;42(5): 905-912.

10. Smith CA, Galante JM, Pierce JL, Scherer LA. Laparoscopic transdiaphragmatic pericardial window: getting to the heart of the matter. Am Coll Surg 2011 Dec;213(6):736-742.

11. Morales $\mathrm{CH}$, Salinas $\mathrm{CM}$, Henao CA, Patiño PA, Mũnoz C. Thoracoscopic pericardial window and penetrating cardiac trauma. J Trauma 1997 Feb;42(2):273-275.

12. Smith CA, Galante JM, Pierce JL, Scherer LA. Laparoscopic transdiaphragmatic pericardial window: getting to the heart of the matter. J Am Coll Surg [Internet] 2011 Dec;213(6): 736-742. Available from: http://dx.doi.org/10.1016/j.jamcollsurg.2011.09.005

13. Cook CC, Gleason TG. Great vessel and cardiac trauma. Surg Clin North Am 2009 Aug;89(4):797-820.

14. Fraga GP, Espínola JP, Mantovani M. Pericardial window used in the diagnosis of cardiac injury/ Janela pericárdica no diagnóstico de lesão cardíaca. Acta Cir Bras 2008;23(2):208-215.

15. Cortés F, Buitrago F. Trauma cardíaco. Rev Fac Med UN Col 2002;50(2):98-102.

16. Asensio JA, Soto SN, Forno W, Roldan G, Petrone P, Salim A, Rowe V, Demetriades D. Penetrating cardiac injuries: a complex challenge. Injury 2001 Sep;32(7):533-543.

17. Tayal VS, Beatty MA, Marx JA, Tomaszewski CA, Thomason MH. FAST (focused assessment with sonography in trauma) accurate for cardiac and intraperitoneal injury in penetrating anterior chest trauma. J Ultrasound Med 2004 Apr;23(4):467-472. 


\section{Ventana Pericardica: Abordaje Subxifoideo vs Toracoscopico En Trauma Precordial Abierto}

Los autores presentan un informe de ventanas pericárdicas practicadas en un centro de atención de pacientes traumatizados de alto nivel de complejidad recolectados durante 54 meses. Ellos realizan ventana pericárdica (subxifoidea o por toracoscopia) a pacientes con herida precordial por arma cortopunzante que se encuentran hemodinamicamente estables.

Analizaron dos cohortes comparables: a un grupo se les practicó ventana pericárdica subxifoidea y a otro ventana pericárdica por toracoscopia; sin embargo, no refieren las razones que definieron el tipo de abordaje lo cual puede representar un sesgo muy importante que limita las conclusiones. Como en otros estudios que se ocupan del problema, el desempeño de estas pruebas diagnósticas fue muy bueno y comparable entre ellas.

Sorprende la alta tasa alta de complicaciones, reintervenciones y reingresos; tratándose de procedimientos diagnósticos ellas se deberían acercar a cero. Los autores no describen las causas de mortalidad, pero si ellas estuvieron relacionadas con el procedimiento diagnóstico las cifras presentadas son prohibitivas.

En el presente la prueba de oro para el diagnóstico de heridas cardíacas en pacientes hemodinamicamente estables es la ecografía subxifoidea (FAST) realizada en el servicio de emergencias; practicada por diferente personal entrenado (radiólogos, cirujanos, urgentólogos) tiene un desempeño comparable a las alternativas invasivas (subxifoidea y toracoscópica), no tiene complicaciones y se puede repetir; por tanto, los diferentes grupos de trauma deben preocuparse por la introducción de este recurso en sus instituciones y por recibir el entrenamiento específico.

Los procedimientos diagnósticos ventana pericárdica subxifoidea o por toracoscopia deben reservarse para aquellos pacientes en quienes la ecografía subxifoidea no fue concluyente por limitaciones relacionadas con el paciente (hemo-neumotórax asociado, lesiones de tejidos blandos), con el procedimiento (falta de colaboración del paciente, pobre resolución del equipo o daño) o dependientes del operador (entrenamiento incompleto). Si se define practicar uno de estos procedimientos invasivos se debe optar por el abordaje toracoscópico cuando en la radiografía simple de tórax se demuestran hallazgos anormales (hemoneumotórax) dadas sus ventajas de explorar la cavidad torácica, hacer un diagnóstico preciso y evacuar la sangre de la cavidad pleural.

Carlos Hernando Morales Presidente, Cirugía Universidad de Antioquia, Medellín, Colombia 


\section{INVITED COMMENTARY}

\section{Pericardic Window: Subxiphoid vs Thoracoscopic Approach in Open Precordial Trauma}

The authors present a report of pericardial windows performed in a trauma center of high complexity level collected during 54 months. They perform a pericardial window (subxiphoid or by thoracoscopy) to patients with precordial gunshot wound that are hemodynamically stable.

They analyzed two comparable cohorts: One group underwent a subxiphoid pericardial window and the other pericardial window through thoracoscopy; however, they do not refer to the reasons that defined the type of approach which may represent a very important bias that limits the conclusions. As in other studies that deal with the problem, the performance of these diagnostic tests was very good and comparable between them.

The high rate of complications, reinterventions and readmissions is surprising; in the case of diagnostic procedures, they should be brought closer to zero. The authors do not describe the causes of mortality, but if they were related to the diagnostic procedure, the figures presented are prohibitive.

At present, the gold test for the diagnosis of cardiac wounds in hemodynamically stable patients is the subxiphoid ultrasound (FAST) performed in the emergency service performed by trained personnel, whether they are radiologists, surgeons or emergency medicine physicians. FAST is comparable to invasive alternatives (subxiphoid or thoracoscopic pericardial window), has no cpmplications and may be repeated. Therefore, different trauma groups should be concerned about the introduction of this resource in their institutions and receiving specific training.

Diagnostic procedures for the subxiphoid pericardial window or by thoracoscopy should be reserved for those patients in whom the subxiphoid ultrasonography was not conclusive due to limitations related to the patient (associated hemo-pneumothorax, soft tissue injuries), with the procedure (lack of patient collaboration, poor equipment resolution or damage) or operator dependent (incomplete training). If one of these invasive procedures is defined, one should opt for the thoracoscopic approach when the chest X-ray shows abnormal findings (hemoneumothorax), given its advantages of exploring the chest cavity, making an accurate diagnosis and evacuating blood from the cavity pleural.

Carlos Hernando Morales

Chairman, Surgery

University of Antioquia, Medellín, Colombia 